\title{
Maximal Domains for Fractional Derivatives and Integrals
}

\section{R. Hilfer * and T. Kleiner}

ICP, Fakultät für Mathematik und Physik, Universität Stuttgart, Allmandring 3, 70569 Stuttgart, Germany

* Correspondence: hilfer@icp.uni-stuttgart.de

Received: 11 March 2020; Accepted: 6 June 2020; Published: 6 July 2020

check for updates

\begin{abstract}
The purpose of this short communication is to announce the existence of fractional calculi on precisely specified domains of distributions. The calculi satisfy desiderata proposed above in Mathematics 7, 149 (2019). For the desiderata (a)-(c) the examples are optimal in the sense of having maximal domains with respect to convolvability of distributions. The examples suggest to modify desideratum (f) in the original list.
\end{abstract}

Keywords: fractional derivatives; fractional integrals; fractional calculus

MSC: 26A33; 34A08; 34K37; 35R11; 44A40

A list of six desiderata was recently proposed in [1] for calling families of operators $\left\{D^{\alpha}, I^{\alpha}\right\}$ with family index $\alpha \in \mathbb{I}$ from some index set $\mathbb{I} \subseteq \mathbb{C}$ fractional derivatives $\left(D^{\alpha}\right)$ and fractional integrals $\left(I^{\alpha}\right)$ of order $\alpha \notin \mathbb{N}$. Distributional domains for $\left\{D^{\alpha}, I^{\alpha}\right\}$ seem to require a minor modification of these desiderata.

Multiplication of distributions is ill-defined so that for distributions desideratum (f) (Leibniz rule) requires generalization. A slightly modified list of desiderata might read as follows:

(a) Integrals $I^{\alpha}$ and derivatives $D^{\alpha}$ of fractional order $\alpha$ should be linear operators on linear spaces.

(b) On some subset $\mathrm{G}_{(\mathrm{b})} \subseteq \mathrm{D}\left(I^{\alpha}\right) \cap I^{\beta}\left[\mathrm{D}\left(I^{\beta}\right)\right] \cap \mathrm{D}\left(I^{\alpha+\beta}\right), \mathrm{G}_{(\mathrm{b})} \neq \varnothing, \mathrm{G}_{(\mathrm{b})} \neq\{0\}$ the index law (semigroup property)

$$
\left(I^{\alpha} \circ I^{\beta}\right) f=I^{\alpha+\beta} f
$$

holds true for $\operatorname{Re} \alpha \geq 0$ and $\operatorname{Re} \beta \geq 0$, where $\mathrm{D}\left(I^{\alpha}\right)$ denotes the domain of $I^{\alpha}$.

(c) Restricted to a suitable subset $\mathrm{G}_{(\mathrm{c})} \subseteq \mathrm{D}\left(I^{\alpha}\right)$ of the domain of $I^{\alpha}$ the fractional derivatives $D^{\alpha}$ of order $\alpha$ operate as left inverses

$$
D^{\alpha} \circ I^{\alpha}=1_{\mathrm{G}_{(\mathrm{c})}}
$$

for all $\alpha$ with $\operatorname{Re} \alpha \geq 0$, where $\circ$ denotes composition of operators, and $1_{\mathrm{G}_{(\mathrm{c})}}$ is the identity on $\mathrm{G}_{(\mathrm{c})}$.

(d) Each of the two limits

$$
\begin{array}{ll}
g^{1}=D^{1} f=\lim _{\alpha \rightarrow 1} D^{\alpha} f, & f \in \mathrm{G}_{(\mathrm{d})}, \\
g^{0}=D^{0} f=\lim _{\alpha \rightarrow 0} D^{\alpha} f, & f \in \mathrm{G}_{(\mathrm{d})}
\end{array}
$$

should exist in some sense on some set $\mathrm{G}_{(\mathrm{d})} \subseteq \mathrm{D}\left(D^{\alpha}\right), \mathrm{G}_{(\mathrm{d})} \neq \varnothing, \mathrm{G}_{(\mathrm{d})} \neq\{0\}$. Moreover, the limiting maps $D^{1}: \mathrm{G}_{(\mathrm{d})} \rightarrow \mathrm{G}_{(\mathrm{d})}$ and $D^{0}: \mathrm{G}_{(\mathrm{d})} \rightarrow \mathrm{G}_{(\mathrm{d})}$ should be linear. 
(e) $\quad D^{0}=1_{\mathrm{G}_{(\mathrm{d})}}$ is the identity on $\mathrm{G}_{(\mathrm{d})}$, i.e., $g^{0}=f$ in Equation (3b).

(f) Endowed with a suitable multiplication $\odot: \mathrm{G}_{(\mathrm{f})} \times \mathrm{G}_{(\mathrm{d})} \rightarrow \mathrm{G}_{(\mathrm{d})}$ the limiting map $D^{1}=D$ obeys the Leibniz rule

$$
D(f \odot g)=f \odot(D g)+(D f) \odot g
$$

for all $f \in \mathrm{G}_{(\mathrm{f})}, g \in \mathrm{G}_{(\mathrm{d})}$ with $\mathrm{G}_{(\mathrm{f})} \neq \varnothing, \mathrm{G}_{(\mathrm{f})} \neq\{0\}$. If $\mathrm{G}_{(\mathrm{d})}$ consist of numerical functions, then $\odot$ is pointwise multiplication and $\mathrm{G}_{(\mathrm{f})}=\mathrm{G}_{(\mathrm{d})}$.

Given these modified desiderata, the objective in this short note is to introduce fractional calculi for distributions. Let us stress that the distributional domains $\mathrm{D}\left(I^{\alpha}\right), \mathrm{D}\left(D^{\alpha}\right)$ given in Theorem 1 below are maximal in a precise mathematical sense. One cannot enlarge them without violating either the desiderata or the interpretation of fractional derivatives and integrals as convolution operators. Recall that numerous other mathematical interpretations exist [2], that may have different maximal domains. In this paper fractional operators are interpreted as convolutions with power law kernels (cf. [2], Equation (28)). A comprehensive analysis of convolutions with power law kernels on weighted spaces of continuous functions was recently given in [3].

Define the spaces of continuously differentiable functions, test functions, and smooth functions with bounded derivates

$$
\begin{aligned}
\mathcal{C}^{m}\left(\mathbb{R}^{d}\right) & :=\left\{f: \mathbb{R}^{d} \rightarrow \mathbb{C} \mid f \text { is } m \text {-times continuously differentiable }\right\} \\
\mathcal{D}\left(\mathbb{R}^{d}\right) & :=\left\{f \in \mathcal{C}^{\infty}\left(\mathbb{R}^{d}\right) \mid f \text { has compact support }\right\} \\
\mathcal{B}\left(\mathbb{R}^{d}\right) & :=\left\{f \in \mathcal{C}^{\infty}\left(\mathbb{R}^{d}\right) \mid f \text { has bounded derivatives }\right\}
\end{aligned}
$$

in the usual way [4]. The spaces $\mathcal{C}^{m}, \mathcal{D}$ are endowed with the norm $\|f\|_{\infty}=\sup |f|$. The topology on $\mathcal{B}$ is induced by the seminorms $\|f\|_{N, g}=\sup \left\{\left\|g \partial^{n_{1}} \ldots \partial^{n_{d}} f\right\|_{\infty}: n_{i} \in \mathbb{N}, \sum_{i}^{d} n_{i} \leq N\right\}$ with $N \in \mathbb{N}$ and $g \in \mathcal{C}_{\mathrm{V}}$, where $\mathcal{C}_{\mathrm{V}}$ is the space of continuous functions vanishing at infinity.

The space of distributions $\mathcal{D}^{\prime}$ is the topological dual of $\mathcal{D}$. The dual space $\mathcal{B}^{\prime}$ is the space of integrable distributions. The pairing $\mathcal{D} \times \mathcal{D}^{\prime} \rightarrow \mathbb{C}$ is denoted $\langle\cdot, \cdot\rangle$, the pairing $\mathcal{B} \times \mathcal{B}^{\prime} \rightarrow \mathbb{C}$ as $\langle\cdot, \cdot\rangle_{\mathcal{B}}$.

Definition 1. Two distributions $f_{1}, f_{2} \in \mathcal{D}^{\prime}\left(\mathbb{R}^{d}\right)$ are called convolvable iff $\varphi\left(f_{1} \otimes f_{2}\right) \in \mathcal{B}^{\prime}\left(\mathbb{R}^{2 d}\right)$ for all $\phi \in \mathcal{D}\left(\mathbb{R}^{d}\right)$, where $\varphi\left(x_{1}, x_{2}\right)=\phi\left(x_{1}+x_{2}\right)$. Their convolution $f_{1} * f_{2}$ is defined by requiring that

$$
\left\langle\phi, f_{1} * f_{2}\right\rangle=\left\langle 1, \varphi\left(f_{1} \otimes f_{2}\right)\right\rangle_{\mathcal{B}}
$$

holds for all $\phi \in \mathcal{D}\left(\mathbb{R}^{d}\right)$.

Let $\mathcal{D}_{+}^{\prime}$ denote the space of causal distributions defined as elements $f \in \mathcal{D}^{\prime}(\mathbb{R})$ whose support is bounded on the left.

Definition 2. Fractional integrals $I_{+}^{\alpha}$ and derivatives $D_{+}^{\alpha}$ are defined for all $\alpha \in \mathbb{C}$ and all distributions $f \in \mathcal{D}_{+}^{\prime}$ as convolution operators

$$
\begin{aligned}
I_{+}^{\alpha} f & :=K_{\alpha} * f \\
D_{+}^{\alpha} f & :=K_{-\alpha} * f
\end{aligned}
$$


with kernels

$$
\begin{array}{ll}
K_{\alpha}(x)= \begin{cases}\frac{x^{\alpha-1}}{\Gamma(\alpha)} & \text { for } x>0 \\
0 & \text { for } x \leq 0\end{cases} \\
K_{\alpha}(x)=\frac{\mathrm{d}^{m}}{\mathrm{~d} x^{m}} K_{\alpha+m}(x) & \text { for } \operatorname{Re} \alpha>0
\end{array}
$$

The operators $I_{+}^{\alpha}$ and $D_{+}^{\alpha}$ are linear and continuous on $\mathcal{D}_{+}^{\prime}$. The kernels $\left\{K_{\alpha}: \alpha \in \mathbb{C}\right\}$ form a convolution group

$$
K_{\alpha} * K_{\beta}=K_{\alpha+\beta}
$$

for all $\alpha, \beta \in \mathbb{C}$. This entails the index law $I_{+}^{\alpha}\left(I_{+}^{\beta} f\right)=I_{+}^{\alpha+\beta} f$ for all $f \in \mathcal{D}_{+}^{\prime}$ and $\alpha, \beta \in \mathbb{C}$. Clearly, all desiderata are fulfilled for $\left\{I_{+}^{\alpha}, D_{+}^{\alpha}\right\}$ with $\mathrm{D}\left(I_{+}^{\alpha}\right)=\mathrm{D}\left(D_{+}^{\alpha}\right)=\mathrm{G}_{(\mathrm{b})}=\mathrm{G}_{(\mathrm{c})}=\mathrm{G}_{(\mathrm{d})}=\mathcal{D}_{+}^{\prime}$ and $\mathrm{G}_{(\mathrm{f})}=\mathcal{C}^{\infty}$.

The domain $\mathcal{D}_{+}^{\prime}$ of causal distributions will now be enlarged using certain sets of lower semicontinuous functions as convolution weights. A function $f: \mathbb{R} \rightarrow \overline{\mathbb{R}}_{+}$, where $\overline{\mathbb{R}}_{+}:=[0, \infty]$, is called lower semicontinuous, if the set $\{f \leq a\}$ is closed for every $a \in \overline{\mathbb{R}}_{+}$. The set of all lower semicontinuous functions is denoted $\mathcal{I}$, the set of lower semicontinuous functions whose support is bounded on the left is denoted $\mathcal{I}_{+}$. For $(p, k) \in \mathbb{R} \times \mathbb{N}$ let

$$
P^{p ; k}:=\left\{f \in \mathcal{I} \mid \exists C>0 \forall x \in \mathbb{R}: f(x) \leq C(1+|x|)^{p}[\log (e+|x|)]^{k}\right\}
$$

be the set of lower semicontinuous functions of power-logarithmic growth of order $(p, k)$. Then

$$
\begin{aligned}
& P_{+}:=\mathcal{I}_{+} \cap\left(\bigcup_{q \in \mathbb{R}} P^{q ; 0}\right) \\
& R_{+}:=\mathcal{I}_{+} \cap\left(\bigcup_{k \in \mathbb{N}_{0}} P^{-1 ; k}\right)
\end{aligned}
$$

are the sets of interest.

Definition 3. Let $U \subseteq \mathcal{D}^{\prime}$ and let $\mathscr{B}(\mathcal{D})$ denote the set of all bounded subsets of $\mathcal{D}$. Then

$$
(U)_{\mathcal{D}^{\prime}}^{*}:=\left\{f \in \mathcal{D}^{\prime}:(f, g) \text { are convolvable for all } g \in U\right\}
$$

denotes the set of all distributions convolvable with the given set $U$. A locally convex topology $\mathscr{T}_{U}$ on $U \subseteq \mathcal{D}^{\prime}$ is defined by the family of seminorms

$$
\|f\|_{V, g}=\left(|f|_{V} *|g|_{V}\right)(0)=\int|f|_{V}(x)|g|_{V}(-x) \mathrm{d} x
$$

with $V \subset \mathcal{D}, V \in \mathscr{B}(\mathcal{D})$ and $g \in(U)_{\mathcal{D}^{\prime}}^{*}$. Here, the $V$-modulus of an element $f \in \mathcal{D}^{\prime}$ is defined as

$$
|f|_{V}(x):=\sup _{g \in V}|\langle f(\cdot), g(\cdot-x)\rangle|
$$

for all $x \in \mathbb{R}$. 
Theorem 1. The convolution group $\left\{K_{\alpha}: \alpha \in \mathbb{C}\right\}$, resp. $\left\{K_{\alpha}: \alpha \in \mathrm{i} \mathbb{R}\right\}$, can be extended from $\left(\mathcal{D}_{+}^{\prime}, \mathscr{T}_{\mathcal{D}_{+}^{\prime}}\right)$ to operate linearly, bijectively, and continuously on the space $\left(U, \mathscr{T}_{U}\right)$ with $U=\left(P_{+}\right)_{\mathcal{D}^{\prime}}^{*}$, resp. $U=\left(R_{+}\right)_{\mathcal{D}^{\prime}}^{*}$ in such a way that compact sets of indices $\alpha$ map to equicontinuous sets of operators.

Corollary 1. The desiderata (a)-(e) are fulfilled for $\left\{I_{+}^{\alpha}, D_{+}^{\alpha}\right\}_{\alpha \in \mathbb{C}}$ with

$$
\mathrm{D}\left(I_{+}^{\alpha}\right)=\mathrm{D}\left(D_{+}^{\alpha}\right)=\mathrm{G}_{(b)}=\mathrm{G}_{(c)}=\mathrm{G}_{(d)}=\left(P_{+}\right)_{\mathcal{D}^{\prime}}^{*}
$$

and for $\left\{I_{+}^{\alpha}, D_{+}^{\alpha}\right\}_{\alpha \in \mathrm{i} \mathbb{R}}$ with $D^{1}, \mathrm{G}_{(d)}$ as in (15a) and

$$
\mathrm{D}\left(I_{+}^{\alpha}\right)=\mathrm{D}\left(D_{+}^{\alpha}\right)=\mathrm{G}_{(b)}=\mathrm{G}_{(c)}=\left(R_{+}\right)_{\mathcal{D}^{\prime}}^{*}
$$

In both cases it is possible to choose $\mathrm{G}_{(f)}=\mathcal{B}$.

The proof of Theorem 1 and its corollary will be published elsewhere, because it is lengthy and giving it here would distract attention from the main message. The domains $D\left(I_{+}^{\alpha}\right), D\left(D_{+}^{\alpha}\right), \mathrm{G}_{(\mathrm{b})}, \mathrm{G}_{(\mathrm{c})}$ are maximal with respect to convolvability in both cases. The second case $\left\{I_{+}^{\alpha}, D_{+}^{\alpha}\right\}_{\alpha \in \mathrm{i}}$ yields a (purely imaginary) "fractional calculus of order zero" in the sense that $\operatorname{Re} \alpha=0$ for all operators in that subset.

\section{References}

1. Hilfer, R.; Luchko, Y. Desiderata for Fractional Derivatives and Integrals. Mathematics 2019, 7, 149. [CrossRef]

2. Hilfer, R. Mathematical and physical interpretations of fractional derivatives and integrals. In Handbook of Fractional Calculus with Applications: Basic Theory; Kochubei, A., Luchko, Y., Eds.; Walter de Gruyter GmbH: Berlin, Germany, 2019; Volume 1, pp. 47-86.

3. Kleiner, T.; Hilfer, R. Weyl Integrals on Weighted Spaces. Fract. Calc. Appl. Anal. 2019, 22, 1225-1248. [CrossRef]

4. Schwartz, L. Theorie des Distributions; Hermann: Paris, France, 1966.

(c) 2020 by the authors. Licensee MDPI, Basel, Switzerland. This article is an open access article distributed under the terms and conditions of the Creative Commons Attribution (CC BY) license (http:// creativecommons.org/licenses/by/4.0/). 